RUP-19-24

\title{
Effect of a second compact object on stable circular orbits
}

\author{
Keisuke Nakashi ${ }^{1, *}$ and Takahisa Igata ${ }^{1, \dagger}$ \\ ${ }^{1}$ Department of Physics, Rikkyo University, Toshima, Tokyo 171-8501, Japan
}

(Dated: June 28, 2021)

\begin{abstract}
We investigate how stable circular orbits around a main compact object appear depending on the presence of a second one by using the Majumudar-Papapetrou dihole spacetime, which consists of the two extremal Reissner-Nordström black holes with different masses. While the parameter range of the separation of the two objects is divided due to the appearance of stable circular orbits, this division depends on its mass ratio. We show that the mass ratio range separates into four parts, and we find three critical values as the boundaries.
\end{abstract}

\section{INTRODUCTION}

Recent progress in the observation of gravitational waves supports the existence of binary black hole systems. The LIGO Scientific and Virgo collaborations have already detected gravitational waves ten times from binary black hole mergers and once from a binary neutron star merger so far [1-4]. Furthermore, since they have started the third observation run, the number of detections will increase in the future. These results imply that binary black hole systems are a quite common phenomenon in our Universe.

A pure binary black hole system is a highly idealized model, around which a third object or matter distribution usually exists in realistic situations. Therefore, one of the next issues is clarifying perturbative interactions with a third body around them. As traditional problems in Newtonian gravity, there are Poincaré's three-body problem and the Kozai mechanism. In recent years, some problems related to these topics have been considered in the framework of the relativistic three body problem $[5,6]$, in the context of resonance in a compound extreme mass ratio inspiral/massive black hole binary [7], and in gravitational wave emission induced by a third body [8-11]. If a third body itself is the target of observation, we can view it as a test body in a fixed background. As a traditional problem in Newtonian gravity, there is Euler's three-body problem, test particle motion in two fixed centers. The corresponding relativistic system is the main topic of this paper.

The study of test body motion is significant for the predictions of astrophysical phenomena around a gravitational system such as a binary. In particular, the circular orbit of a test body plays some essential roles in both theory and observations. In the black hole spacetime, for instance, the bending of light due to strong gravity makes a photon orbit circular near the horizon. If the circular photon orbit is unstable, it relates to the formation of the black hole shadow. On the other hand, the sequence of stable circular massive particle orbits is relevant to accretion disks and a binary system. The innermost stable circular orbit (ISCO) radius is a distinctive one because it is identified as the inner edge of a standard accretion disk model and a compact binary switches the stage of the evolution from the inspiraling phase to the merging phase there $[12,13]$.

Actual binary black hole systems exist as highly dynamical systems so that one needs to use the numerical method to analyze the phenomena around such systems; for example, the study of the shadow of a binary black hole system requires a fully nonlinear analysis of the numerical relativity [14]. On the other hand, it is also significant to use an analytical method for a qualitative understanding. To this end, we often employ some axisymmetric and stationary (or static) dihole spacetime as a toy model. There are some exact dihole spacetime solutions of the Einstein equation (or the Einstein-Maxwell equation) such as the Weyl spacetime [15], the Majumdar-Papapetrou spacetime [16-18], the double-Kerr spacetime [19], etc. We can extract the specific features of phenomena around a binary black hole system by using these dihole spacetimes. Indeed, the eyebrows structure of the binary black hole shadow is reproduced in the (quasi)static dihole spacetime [20-24].

The aim of the present paper is to reveal how the marginally stable circular orbit (MSCO) or ISCO of the dihole spacetime varies compared to those of the single black hole spacetime. To achieve this, we adopt the MajumdarPapapetrou (MP) dihole spacetime, which contains two extremal Reissner-Nordström black holes. The circular orbit and its stabilities in the equal mass MP dihole spacetime have been investigated [25-27]. In our previous paper [25], we clarified the dependence of the positions of MSCOs and ISCOs on the separation parameter in the equal mass MP

\footnotetext{
*Electronic address: nakashi@rikkyo.ac.jp
}

${ }^{\dagger}$ Electronic address: igata@rikkyo.ac.jp 
dihole spacetime. We found that the range of the dihole separation is divided into five ranges and obtained the four critical values as the boundaries. In this paper, we investigate the sequence of the stable circular orbit in the different mass MP dihole spacetime, which consists of the two different mass extremal Reissner-Nordström black holes. Once we fix the mass scale of one of the two black holes, the system depends on two parameters: the separation and the mass ratio. As the result of our analysis, we divide the mass ratio parameter range into four ranges and obtain three critical values as the boundaries.

This paper is organized as follows. In the following section, we introduce the MP dihole spacetime with different masses and derive conditions for circular particle orbits on the background. Furthermore, we clarify the stability conditions of these orbits in terms of the Hessian of a $2 \mathrm{D}$ potential function. In Sec. III, while changing the mass ratio of the dihole, we analyze the dihole separation dependence of the positions of stable circular orbits. Due to some qualitative differences of sequences of stable circular orbits, we classify the range of dihole mass ratios into four parts and determine three critical values of the mass ratio as the boundaries of the range. Section IV is devoted to a summary and discussions. Throughout this paper, we use units in which $G=1$ and $c=1$.

\section{CONDITIONS FOR STABLE CIRCULAR ORBITS IN THE MAJUMDAR-PAPAPETROU DIHOLE SPACETIME}

The metric and the gauge field of the MP dihole spacetime in isotropic coordinates are given by

$$
\begin{aligned}
& g_{\mu \nu} \mathrm{d} x^{\mu} \mathrm{d} x^{\nu}=-\frac{\mathrm{d} t^{2}}{U^{2}}+U^{2}\left(\mathrm{~d} \rho^{2}+\rho^{2} \mathrm{~d} \phi^{2}+\mathrm{d} z^{2}\right), \\
& A_{\mu} \mathrm{d} x^{\mu}=U^{-1} \mathrm{~d} t, \\
& U(\rho, z)=1+\frac{M_{+}}{\sqrt{\rho^{2}+(z-a)^{2}}}+\frac{M_{-}}{\sqrt{\rho^{2}+(z+a)^{2}}},
\end{aligned}
$$

where $M_{ \pm}$are each black hole mass located at $z= \pm a(a \geq 0)$. We introduce a mass ratio parameter

$$
\nu:=\frac{M_{-}}{M_{+}} .
$$

Without loss of generality, we assume that the black hole with mass $M_{+}$is larger than that with mass $M_{-}$, i.e.,

$$
0 \leq \nu \leq 1
$$

We use units in which $M_{+}=1$ in what follows.

The Lagrangian of a particle freely falling in the MP dihole spacetime is given by

$$
\mathscr{L}=\frac{1}{2}\left[-\frac{\dot{t}^{2}}{U^{2}}+U^{2}\left(\dot{\rho}^{2}+\rho^{2} \dot{\phi}^{2}+\dot{z}^{2}\right)\right],
$$

where the dot denotes derivative with respect to an affine parameter. Since the coordinates $t$ and $\phi$ are cyclic, the canonical momenta conjugate to them are constants of motion:

$$
E=\frac{\dot{t}}{U^{2}}, \quad L=\rho^{2} U^{2} \dot{\phi}
$$

which are energy and angular momentum, respectively. We normalize the 4 -velocity $\dot{x}^{\mu}$ so that $g_{\mu \nu} \dot{x}^{\mu} \dot{x}^{\nu}=-\kappa$, where $\kappa=1$ for a massive particle and $\kappa=0$ for a massless particle. Rewriting the normalization condition in terms of $E$ and $L$, we have

$$
\begin{aligned}
& \dot{\rho}^{2}+\dot{z}^{2}+V=E^{2}, \\
& V(\rho, z)=\frac{L^{2}}{\rho^{2} U^{4}}+\frac{\kappa}{U^{2}} .
\end{aligned}
$$

We can view Eq. (8) as an energy equation and $V$ as a $2 \mathrm{D}$ effective potential of particle motion in the $\rho-z$ plane. In terms of $V$, the equations of motion are written as

$$
\begin{aligned}
& \ddot{\rho}+\frac{2 U_{z}}{U} \dot{z} \dot{\rho}-\frac{2 U_{\rho}}{U} \dot{z}^{2}+\frac{V_{\rho}}{2}=0, \\
& \ddot{z}+\frac{2 U_{\rho}}{U} \dot{z} \dot{\rho}-\frac{2 U_{z}}{U} \dot{\rho}^{2}+\frac{V_{z}}{2}=0,
\end{aligned}
$$


where $V_{i}=\partial_{i} V$ and $U_{i}=\partial_{i} U(i=\rho, z)$.

We focus on circular orbits with constant $\rho$ and $z$. Then, the energy equation (8) immediately reduces to

$$
V=E^{2} \text {. }
$$

Hence, $V$ must be positive for circular orbits. In addition, we find that constant $(\rho, z)$ can be a solution to Eqs. (10) and (11) when its position corresponds to an extremum of $V$ :

$$
\begin{aligned}
& V_{\rho}=0, \\
& V_{z}=0 .
\end{aligned}
$$

We can rewrite the three conditions (12)-(14), respectively, as

$$
\begin{aligned}
& E^{2}=E_{0}^{2}(\rho, z):=V\left(\rho, z ; L_{0}^{2}\right), \\
& L^{2}=L_{0}^{2}(\rho, z):=-\frac{\rho^{3} U^{2} U_{\rho}}{U+2 \rho U_{\rho}}, \\
& U_{z}=\frac{a-z}{\left[\rho^{2}+(z-a)^{2}\right]^{3 / 2}}-\frac{\nu(a+z)}{\left[\rho^{2}+(z+a)^{2}\right]^{3 / 2}}=0 .
\end{aligned}
$$

From Eqs. (15) and (16), both values of $E_{0}^{2}$ and $L_{0}^{2}$ depend on positions of circular orbits and must be positive. The positivity of $L^{2}$ leads to that of $E^{2}$ as seen from Eq. (9), so that it is sufficient to pay attention only to the positivity of $L^{2}$.

Now we solve Eq. (17). If $\nu=0$, then we obtain the solution $z=a$. If $0<\nu \leq 1$, we find from Eq. (17) that the range of $z$ is bounded in $|z|<a$. Solving it for $\rho^{2}$ in this range, we obtain the root

$$
\rho_{0}^{2}(z)=\frac{(a-z)^{2 / 3}(a+z)^{2}-\nu^{2 / 3}(a+z)^{2 / 3}(a-z)^{2}}{\nu^{2 / 3}(a+z)^{2 / 3}-(a-z)^{2 / 3}}, \quad z \neq \frac{1-\nu}{1+\nu} a .
$$

When $z=a(1-\nu) /(1+\nu)$ holds, then Eq. (17) leads to $z=0$, and hence $\nu=1$. Note that the root $\rho_{0}$ is real and positive in the range

$$
-a<z<-\frac{1-\sqrt{\nu}}{1+\sqrt{\nu}} a, \quad \frac{1-\nu}{1+\nu} a<z<a .
$$

The curve $\rho=\rho_{0}$ asymptotically approaches the line

$$
z=\frac{1-\nu}{1+\nu} a .
$$

In particular, the intersection point of the line with the symmetric axis $\rho=0$ corresponds to the center of mass of the dihole. On the other hand, the curves terminate on $\rho=0$ at $z= \pm a$ (i.e., the horizons) and

$$
z=-\frac{1-\sqrt{\nu}}{1+\sqrt{\nu}} a .
$$

Therefore, we can find a circular orbit at a point in the $\rho$ - $z$ plane if it is located on the curve $\rho=\rho_{0}(z)$ and satisfies $E_{0}^{2} \geq 0$ and $L_{0}^{2} \geq 0$.

To determine the stability of a circular orbit, we need further analysis. We consider the linear stability of circular particle motion in terms of the Hessian $V_{i j}$, where $V_{i j}=\partial_{j} \partial_{i} V(i, j=\rho, z)$. Let $h$ be its determinant, $h\left(\rho, z ; L^{2}\right)=$ $\operatorname{det} V_{i j}$, and $k$ be its trace, $k\left(\rho, z ; L^{2}\right)=\operatorname{tr} V_{i j}$. By using these we define the region $D$ in the $\rho-z$ plane by

$$
D=\left\{(\rho, z) \mid L_{0}^{2}>0, h_{0}>0, k_{0}>0\right\},
$$

where

$$
\begin{aligned}
& h_{0}(\rho, z)=\left.h\left(\rho, z ; L_{0}^{2}\right)\right|_{U_{z}=0}, \\
& k_{0}(\rho, z)=\left.k\left(\rho, z ; L_{0}^{2}\right)\right|_{U_{z}=0},
\end{aligned}
$$

where the restriction $U_{z}=0$ means to eliminate the terms proportional to $U_{z}$. We can find stable circular orbits on the curve $\rho=\rho_{0}(z)$ included in the region $D$. 


\section{DEPENDENCE OF THE SEQUENCE OF STABLE CIRCULAR ORBITS ON THE MASS RATIO}

In this section, focusing on stable circular orbits in the MP dihole spacetime, we analyze the dependence of sequences of their orbits on the separation $a$ for various values of the mass ratio $\nu$.

\section{A. $\quad \nu=1$}

In the beginning, let us recall how sequences of stable circular orbits change as the separation $a$ varies in the equal unit mass MP dihole spacetime (i.e., $\nu=1$ and $M_{+}=M_{-}=1$ ) [25]. For $a>a_{0}=1.401 \cdots$, a sequence of stable circular orbits exists in the range $\rho \in(\sqrt{2} a, \infty)$ on the equidistant symmetric plane $z=0$ from each black hole. Furthermore, it bifurcates at $(\rho, z)=(\sqrt{2} a, 0)$ and extends towards each black hole. As a result, we have three MSCOs, two of which are the ISCOs. At $a=a_{0}$, the three MSCOs degenerate at $(\rho, z)=\left(\sqrt{2} a_{0}, 0\right)$. For $a_{0} \geq a>a_{*}=0.9713 \cdots$, a single sequence of stable circular orbits appears on $z=0$ in the range $\rho \in(\sqrt{2} a, \infty)$, and this inner boundary corresponds to the ISCO. At $a=a_{*}$, the single sequence is marginally connected at a point where $h_{0}$ has a saddle point. For $a_{*} \geq a>a_{\mathrm{c}}=0.3849 \cdots$, we have two sequences of stable circular orbits on $z=0$. This phenomenon implies the possibility of double accretion disk formation in this system. In particular, for $a_{*}>a>a_{\infty}=0.5433 \cdots$, the outer sequence exists from infinity to an MSCO and the inner sequence from an MSCO to the ISCO, while for $a_{\infty} \geq a>a_{\mathrm{c}}$, the outer boundary of the inner sequence is no longer a marginally stable circular massive particle orbit but turns into a circular photon orbit; that is, infinitely large energy would be required for the stable circular orbit. At $a=a_{\mathrm{c}}$, the inner sequence just disappears. For $a_{\mathrm{c}} \geq a \geq 0$, we only have a single sequence of stable circular orbits from infinity to the ISCO on $z=0$.

In the following subsections, dividing the range of $\nu$ into four parts, we consider the dependence of sequences of stable circular orbits on the separation $a$ in each range of $\nu$.

$$
\text { B. } 1>\nu>\nu_{\infty}=0.7698 \cdots
$$

We consider sequences of stable circular orbits for various values of $a$ in the MP dihole spacetime with mass ratio $\nu \simeq 1$ but $\nu \neq 1$. We show sequences of stable circular orbits for several values of $a$ in the case $\nu=0.9$ in Fig. 1 . On the basis of these typical plots, we discuss some qualitative properties of stable circular orbits and critical values of a. Specific numerical values for critical values $a_{0}, a_{*}, a_{\infty}$, and $a_{\mathrm{c}}$ in this subsection are those for $\nu=0.9$.

For a large value of $a$, we have two sequences of stable circular orbits on both sides of the dihole [see Fig. 1(a)]. The sequence on the large black hole side exists from infinity to the ISCO near the large black hole. On the other hand, the sequence on the small black hole side is restricted within a finite region. The inner boundary near the small black hole corresponds to the ISCO, and the outer boundary to an MSCO. As the value of $a$ approaches a critical value $a_{0}(=2.111 \cdots)$ from above, the MSCO and the ISCO on the small black hole side approach each other. At $a=a_{0}$, these merge into one, and then the sequence on the small black hole side just disappears [see Fig. 1(b)]. If $a$ becomes smaller than $a_{0}$, the sequence on the small black hole side no longer exists. We can interpret this disappearance as a consequence of relativistic effects because the corresponding sequences in Euler's three-body system, which is governed by Newtonian gravity, always exist for arbitrary values of $a$.

In the range $a_{0} \geq a>a_{*}(=0.9252 \cdots)$, there exists a sequence of stable circular orbits only on the large black hole side, which appears from infinity to the ISCO [see Fig. 1(c)]. At $a=a_{*}$, the boundary of the region $D$ touches the curve $\rho=\rho_{0}$ [see Fig. 1(d)]. This implies that two sequences of stable circular orbits are marginally connected at a point.

In the range $a_{*} \geq a>a_{\mathrm{c}}(=0.4679 \cdots)$, two sequences of stable circular orbits appear [see Figs. 1(d)-1(g)]. The outer sequence exists from infinity to the outermost MSCO. On the other hand, the behavior of the inner sequence divides this range of $a$ into two parts. For $a_{*} \geq a>a_{\infty}(=0.5198 \cdots)$, the inner sequence exists between an MSCO and the ISCO. However, at $a=a_{\infty}$, the outer MSCO disappears because infinitely large energy and angular momentum would be required for a massive particle [see Fig. 1(f)]. In other words, a circular photon orbit appears there. For $a_{\infty} \geq a>a_{\mathrm{c}}$, the inner sequence appears between the stable circular photon orbit and the ISCO [see Fig. 1(g)]. At $a=a_{\mathrm{c}}$, the stable circular photon orbit and the ISCO merge into one, and then the inner sequence just disappears [see Fig. 1(h)]. In the range $a_{\mathrm{c}} \geq a \geq 0$, there only exists a sequence of stable circular orbits, which appears from infinity to the ISCO [see Fig. 1(i)].

Consequently, we divide the range of $a$ into five parts on the basis of typical behaviors of the sequence of stable circular orbits and introduce four critical values of $a$ as the boundaries of these ranges as we have done in the case 
$\nu=1$. Note that, however, each meaning of critical values is slightly generalized from those of $\nu=1$. Here, let us summarize how we define the four critical values:

(i) $a=a_{0}$ : The sequence of stable circular orbits on the small black hole side disappears.

(ii) $a=a_{*}$ : The sequence of stable circular orbits on the large black hole side is divided into two parts.

(iii) $a=a_{\infty}$ : A stable circular photon orbit appears at the outer boundary of the inner sequence of stable circular orbits on the large black hole side.

(iv) $a=a_{\mathrm{c}}$ : The inner sequence of stable circular orbits on the large black hole side disappears.

In the following, according to the difference in the appearance of these critical values, we classify the range of the mass ratio $\nu$ into four parts. In each range of $\nu$, we discuss the behavior of the sequence of stable circular orbits depending on $a$. Figure 6(a) shows the dependence of the radii of the MSCOs, the ISCOs, and the circular photon orbits on $a$ in the case $\nu=0.9$. In the range $a_{\mathrm{c}}<a \leq a_{\infty}$, the radius of the ISCO (red solid line) is smaller than the one of the stable circular photon orbit (orange solid line in the middle of the three). In addition, the discontinuous transition of the position of the ISCO occurs at $a=a_{\mathrm{c}}$. These phenomena are also seen in the equal mass MP dihole spacetime [25].

$$
\text { C. } \nu=\nu_{\infty}=0.7698 \cdots
$$

If we decrease the value of $\nu$ from $\nu=1$, then at

$$
\nu=\nu_{\infty}:=\frac{4 \sqrt{3}}{9}=0.7698 \cdots,
$$

the stable circular photon orbit no longer appears for any value of $a$. In other words, the critical value $a_{\infty}$ disappears at $\nu=\nu_{\infty}$. We can interpret that the gravity of the small black hole is not sufficiently strong to make a photon orbit circular in the region far from the large black hole even if two black holes get close each other. In what follows, we consider sequences of stable circular orbits in the case $\nu=\nu_{\infty}$.

For $a>1 / 2$, the behavior of sequences of stable circular orbits is similar as that discussed in the previous subsection. Indeed, we find two critical values $a_{0}=2.269 \cdots$ and $a_{*}=0.8740 \cdots$. We note that, however, qualitative differences from the case in the previous subsection appear at $a=1 / 2$. In the limit as $a \searrow 1 / 2$, we find that the MSCO and the ISCO at the boundaries of the inner sequence merge into one at $(\rho, z)=(2 \sqrt{2} / 3,1 / 6)=(0.9428 \cdots, 0.1666 \cdots)$ (see Fig. 2). Simultaneously, infinitely large energy and angular momentum are required for a massive particle to orbit circularly here. In other words, here is a stable/unstable circular photon orbit. These behaviors mean that $a_{\mathrm{c}}$ and $a_{\infty}$ are degenerate at $a=1 / 2$, that is, $a=a_{\mathrm{c}}=a_{\infty}=1 / 2$.

In the range $a<1 / 2$, there is only a single sequence of stable circular orbits that appears from infinity to the ISCO, which is the same as that discussed in the previous section.

$$
\text { D. } \nu_{\infty}>\nu>\nu_{*}=0.5306 \cdots
$$

We consider sequences of stable circular orbits for various values of $a$ in the case where $\nu_{\infty}>\nu>\nu_{*}=0.5306 \cdots$. We can see typical sequences of stable circular orbits for $\nu=0.7$ in Fig. 3 . On the basis of these plots, we discuss the appearance of critical values $a_{0}, a_{*}$, and $a_{\mathrm{c}}$ in this range. Specific numerical values for these critical values in this subsection are those for $\nu=0.7$.

For a relatively large value of $a$, a sequence of stable circular orbits appears from infinity to the ISCO on the large black hole side, while a sequence appears between an MSCO and the ISCO on the small black hole side [see Fig. 3(a)]. When $a$ becomes smaller and smaller, at $a=a_{0}(=2.285 \cdots)$, the sequence on the small black hole side disappears [see Fig. 3(b)]. When $a$ becomes smaller and smaller yet, at $a=a_{*}(=0.8520 \cdots)$, the sequence on the large black hole side is divided into two parts. In the range $a_{*} \geq a>a_{\mathrm{c}}(=0.6454 \cdots)$, there are two sequences, the inner and the outer. As a result, we find three MSCOs as the boundaries of these sequences, and the innermost one corresponds to the ISCO. At $a=a_{\mathrm{c}}$, the inner sequence disappears. Note that the critical value $a_{\infty}$ no longer exists in this range of $\nu$. In the range $0 \leq a<a_{\mathrm{c}}$, we find a single sequence that appears from infinity to the ISCO. The dependence of the radii of the MSCOs, the ISCOs, and the circular photon orbits on $a$ in the case $\nu=0.7$ is shown in Fig. 6(b). The parameter range of $a$ is divided by $a_{0}, a_{*}$, and $a_{\mathrm{c}}$ into four parts. The discontinuous transition of the ISCO on the large black hole side still occurs at $a=a_{\mathrm{c}}$. For any value of $a$, stable circular photon orbits do not exist. 


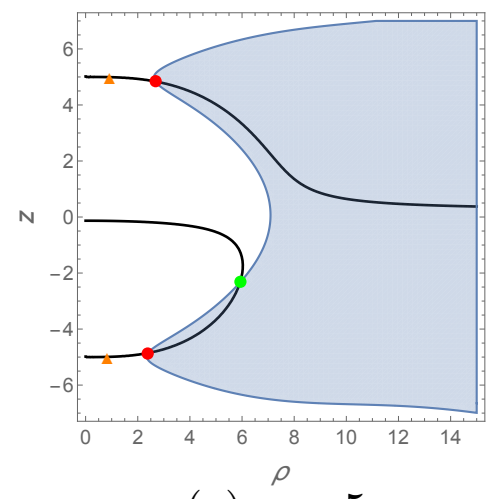

(a) $a=5$

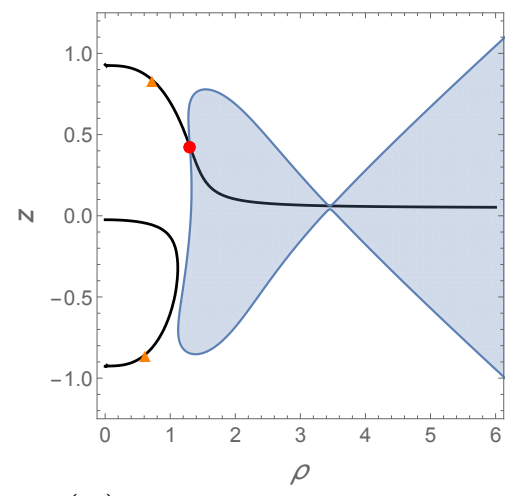

(d) $a=a_{*}=0.9252 \ldots$

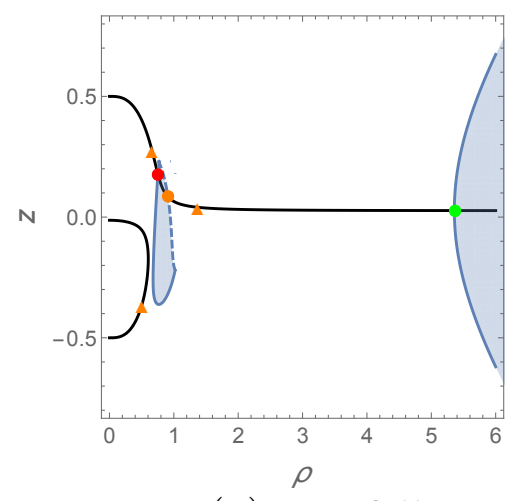

(g) $a=0.5$

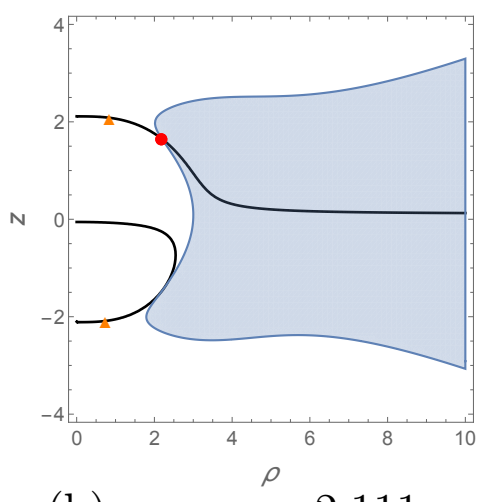

(b) $a=a_{0}=2.111 \cdots$

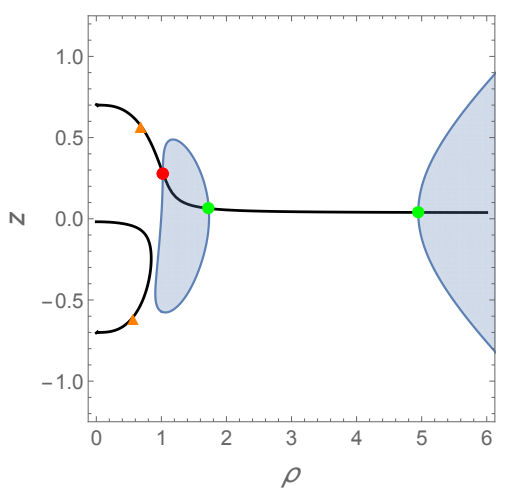

(e) $a=0.7$

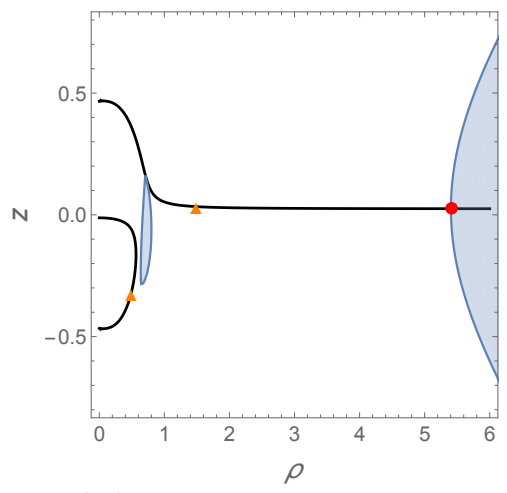

(h) $a=a_{\mathrm{c}}=0.4679 \ldots$

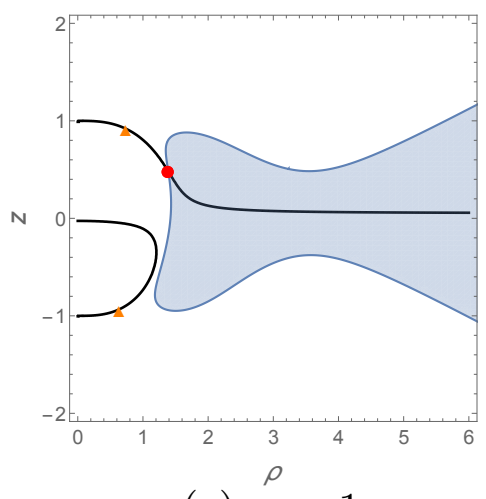

(c) $a=1$

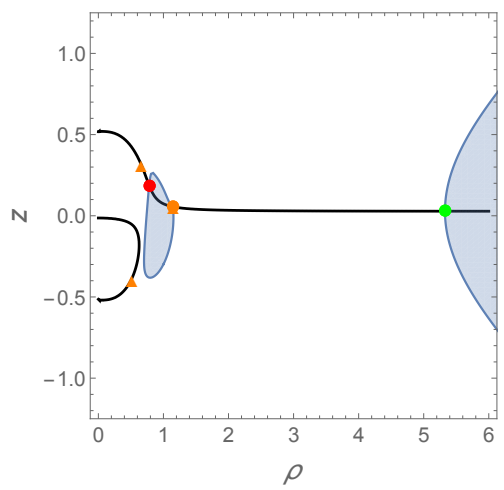

(f) $a=a_{\infty}=0.5198 \ldots$

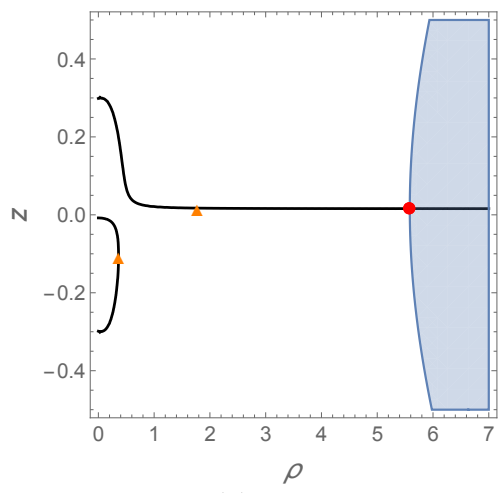

(i) $a=0.3$

FIG. 1: Sequences of stable circular orbits in the Majumdar-Papapetrou dihole spacetime with mass ratio $\nu=0.9$. The black solid lines denote the curve $\rho=\rho_{0}$. The shaded regions denote the region $D$, where $h_{0}>0, L_{0}^{2}>0$, and $k_{0}>0$. The boundaries of $D$ are shown by the blue solid lines on which $h_{0}$ vanishes and the blue dashed lines on which $L_{0}^{2}$ diverges. The black solid lines in the shaded regions show the positions of stable circular orbits. The green dots indicate the position of marginally stable circular orbits, and the red dots indicate the position of the innermost stable circular orbits. The orange dots show the positions of stable circular photon orbits, and the orange triangles show those of unstable ones.

$$
\text { E. } \quad \nu=\nu_{*}=0.5306 \cdots
$$

We focus on sequences of stable circular orbits for various values of $a$ in the case $\nu=\nu_{*}=0.5306 \cdots$. For large $a$, we can see similar behavior of the sequences of stable circular orbits as is shown in the previous subsection. Indeed, we obtain the critical value $a_{0}=2.189 \cdots$. We should note that the inner sequence of stable circular orbits appearing at $(\rho, z)=(2.279 \cdots, 0.3637 \cdots)$ for $a=a_{*}=0.8327 \cdots$ disappears as soon as it appears [see Fig. 4(d)]. This means that the critical values $a_{*}$ and $a_{\mathrm{c}}$ are degenerate. Consequently, we have no inner sequence of stable circular orbits 


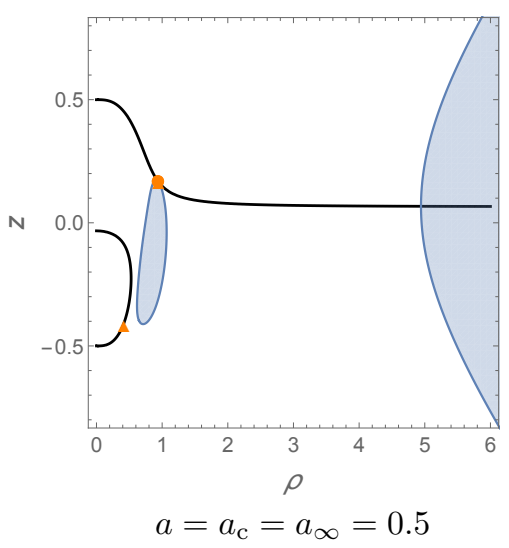

FIG. 2: Sequences of stable circular orbits in the Majumdar-Papapetrou dihole spacetime with mass ratio $\nu=\nu_{\infty}=0.7698 \cdots$. The roles of each element in these plots are the same as those in Fig. 1.

on the large black hole side.

$$
\text { F. } \quad \nu_{*}>\nu>\nu_{0}=0.0110134 \cdots
$$

Let us consider sequences of stable circular orbits for various values of $a$ in the case where $0<\nu<\nu_{*}$. Observing typical sequences for $\nu=0.3$ in Fig. 5 , we discuss the appearance of the critical value $a_{0}$ in this range. The specific numerical value of $a_{0}$ in this subsection is that for $\nu=0.3$.

For a large value of $a$, we find two sequences of stable circular orbits on both sides of the dihole [see Fig. 5(a)]. On the large black hole side, the sequence appears from infinity to the ISCO. On the small black hole side, the sequence appears from the outer MSCO to the ISCO. If $a$ becomes smaller and reaches $a=a_{0}(=1.762 \cdots)$, the sequence on the small black hole side disappears. Therefore, there still exists the critical value $a_{0}$ [see Fig. 5(b)]. In the range $a<a_{0}$, however, any qualitative change of the sequence of stable circular orbits occurs on the large black hole side. The dependence of the radii of the MSCOs, the ISCOs, and the circular photon orbits on $a$ in the case $\nu=0.3$ is shown in Fig. 6(c). The parameter range of $a$ is divided by $a_{0}$ into two parts. The continuous transition of the ISCO on the large black hole side no longer occurs because there are no separated sequences of stable circular orbits on the large black hole side.

$$
\text { G. } 0 \leq \nu \leq \nu_{0}
$$

We mention the sequence of stable circular orbits in the range $0 \leq \nu \leq \nu_{0}$. When the value of $\nu$ reaches $\nu_{0}$ from above, the critical value $a_{0}$ is equal to zero. This means that the sequence on the small black hole side does not vanish unless the two black holes coalesce into one. If we make the value of $\nu$ smaller than $\nu_{0}$, any critical values of $a$ do not appear. According to Fig. 6(d), where we set $\nu=0.01$, for $a>0$, both the large and the small black holes have the sequence of the stable circular orbits; i.e., two MSCOs - one of these is also the ISCO - always appear on the small black hole.

\section{SUMMARY AND DISCUSSIONS}

We have investigated the sequence of stable circular orbits around the symmetric axis in the Majumdar-Papapetrou (MP) dihole spacetime with different masses. Once we fix the mass of the large black hole to 1, the MP dihole spacetime is characterized by two parameters: the separation $a$ and the mass ratio $\nu$.

When $\nu \simeq 1$ but $\nu \neq 1$, the sequence of the stable circular orbits changes as $a$ varies in common with the case of $\nu=1$, but we have generalized the definitions of the critical values of $a$ to be valid for the case of the different mass MP dihole from those in the equal mass MP dihole spacetime [25]. When the value of $a$ is relatively large, the sequence of stable circular orbits on the large black hole side exists from infinity to the ISCO while that on the small black hole side is restricted to a finite range. At $a=a_{0}$, the sequence on the small black hole side disappears. This phenomenon occurs due to the relativistic effect of the appearance of the ISCOs; that is, since the radius of the 


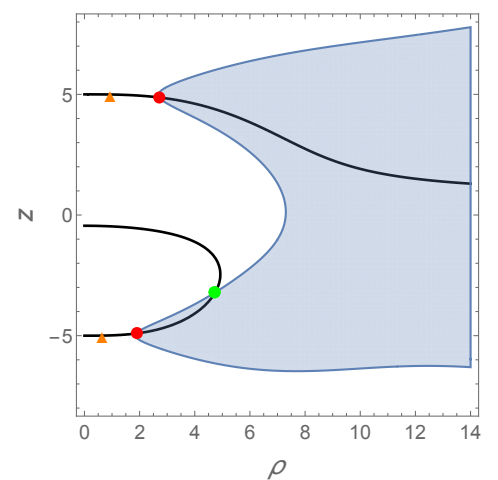

(a) $a=5$

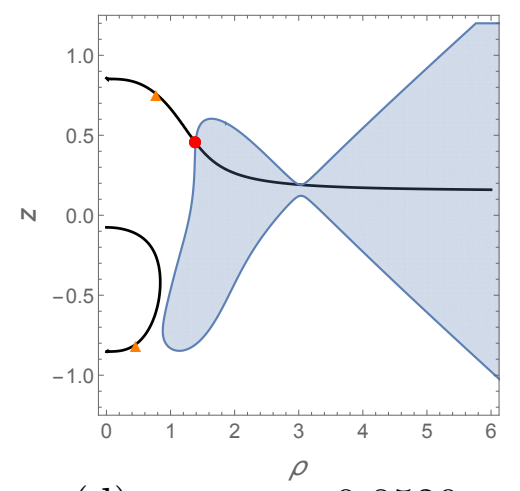

(d) $a=a_{*}=0.8520 \cdots$

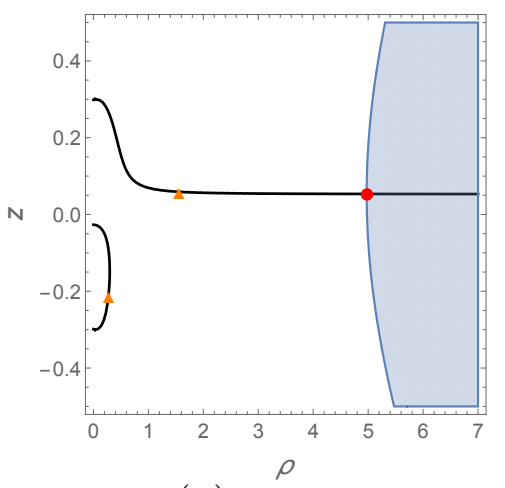

(g) $a=0.3$

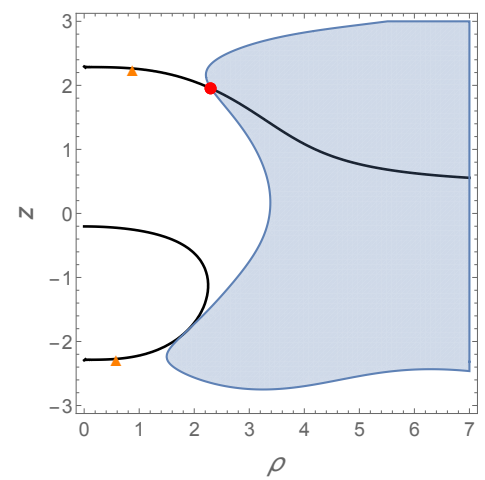

(b) $a=a_{0}=2.285 \cdots$

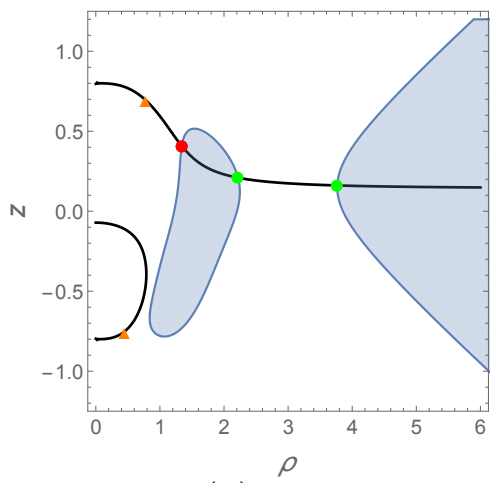

(e) $a=0.8$

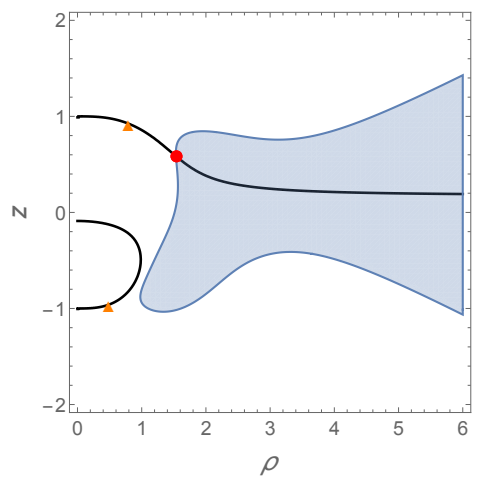

(c) $a=1$

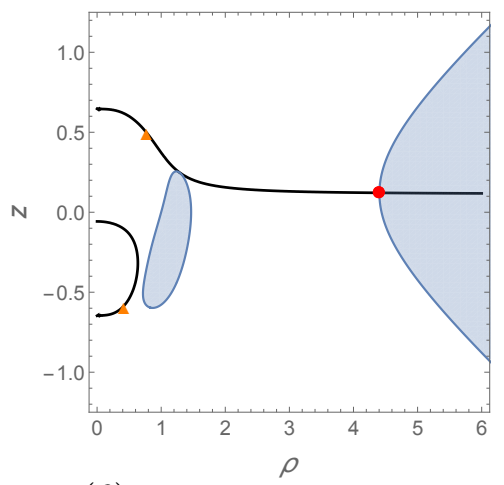

(f) $a=a_{\mathrm{c}}=0.6454 \cdots$

FIG. 3: Sequence of stable circular orbits in the Majumdar-Papapetrou dihole spacetime with mass ratio $\nu=0.7$. The roles of each element in these plots are the same as those in Fig. 1.

outer MSCO on the small black hole side decreases faster than the one of the ISCO as $a$ decreases, the positions of the MSCO and the ISCO coincide with each other at $a=a_{0}$, and then the sequence on the small black hole side disappears [see the green and red dashed lines in Figs. 6(a)-6(c)]. For $a<a_{0}$, the sequence of stable circular orbits appears only on the large black hole side. When $a=a_{*}$, the sequence on the large black hole side is marginally connected at a point. In the range $a_{\mathrm{c}}<a<a_{*}$, two sequences of the stable circular orbits appear on the large black hole side. The outer boundary of the inner sequence is an MSCO in $a_{\infty}<a<a_{*}$ while a stable circular photon orbit in $a_{\mathrm{c}}<a \leq a_{\infty}$. Finally, for $0 \leq a \leq a_{\mathrm{c}}$, since the inner sequence vanishes, we have a single connected sequence from infinity to the ISCO on the large black hole side.

We have also revealed the dependence of the sequence of stable circular orbits on $\nu$. Figure 7 shows the relation between $\nu$ and the critical values of $a$. For $\nu>\nu_{\infty}=0.7698 \cdots$, the sequences of the stable circular orbits are qualitatively the same as these of the case $\nu \simeq 1$. At $\nu=\nu_{\infty}$, the two critical values $a_{\infty}$ and $a_{\mathrm{c}}$ merge with each 


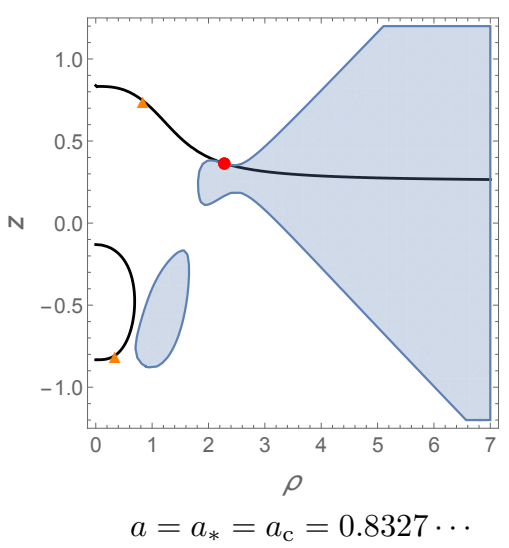

FIG. 4: Sequences of stable circular orbits in the Majumdar-Papapetrou dihole spacetime with mass ratio $\nu=\nu_{*}=0.5306 \cdots$. The roles of each element in this plot are the same as those in Fig. 1.

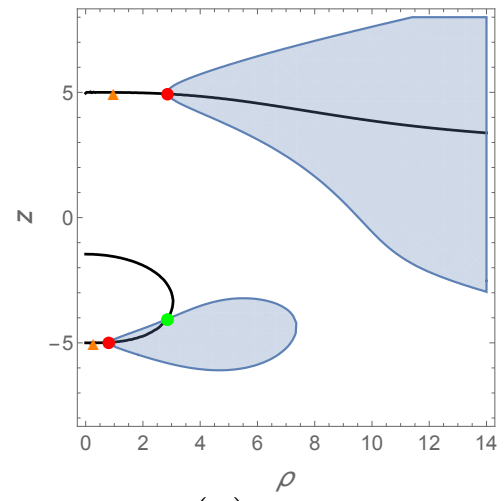

(a) $a=5$

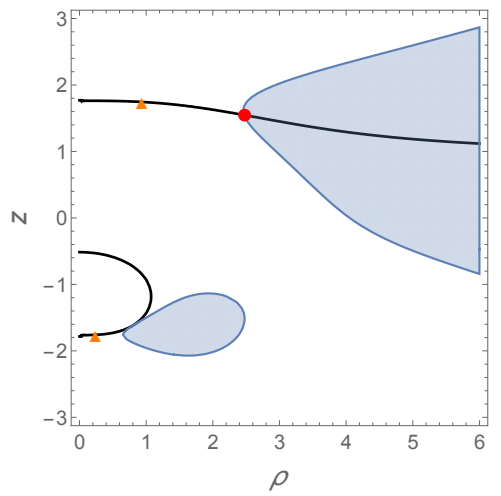

(b) $a=a_{0}=1.762 \cdots$

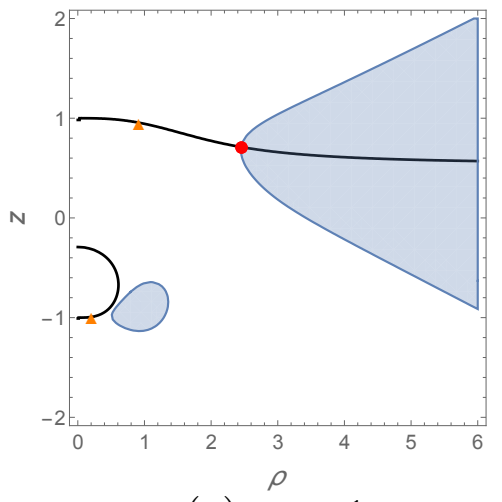

(c) $a=1$

FIG. 5: Sequence of stable circular orbits in the Majumdar-Papapetrou dihole spacetime with mass ratio $\nu=0.3$. The roles of each element in these plots are the same as those in Fig. 1.

other, so that the parameter range of $a$ is divided into four parts. At $\nu=\nu_{*}=0.5306 \cdots$, the critical values $a_{*}$ and $a_{\mathrm{c}}$ coincide with each other. For $\nu<\nu_{*}$, the sequence on the large black hole side does not separate into two parts. The remaining critical value $a_{0}$ also disappears when $\nu=\nu_{0}=0.01101 \cdots$. When we make the value of $\nu$ smaller than $\nu_{0}$, the sequences of stable circular orbits on both sides do not vanish until the two black holes merge into one [see Fig. 6 (d)].

The phenomena we have revealed are not caused by electric charges of the black holes, but by the presence of two black holes. Hence, in our Universe, we can observe such phenomena occurring around a compact object accompanied by a second compact object. The sequence of stable circular orbits is not on a flat plane because of the existence of a second compact object. Therefore, we may observe a deformed accretion disk that indicates the existence of another gravitational source. Furthermore, for any value of $\nu$, the radius of the ISCO on the large black hole side tends to be more inner than the case of a single black hole. This suggests that high energy X-rays can be detected compared to the single black hole case because the effective temperature of the standard disk is higher as the radius of the ISCO is smaller [28, 29]. In the ranges $\nu_{*}<\nu \leq 1$ and $a_{\mathrm{c}}<a<a_{*}$, we may observe double accretion disks. Since a relativistic effect causes the inner disk, we can use it in the testing of gravitational theories. The observation of the accretion disk of a main black hole with the second companion object (e.g., blazar OJ287) may help us to explore the effect of the second compact object [30]. The presence of the stable circular photon orbit in the ranges $\nu_{\infty}<\nu \leq 1$ and $a_{\mathrm{c}}<a \leq a_{\infty}$ is a characteristic property of the dihole spacetime and is associated with distinctive phenomenological features, such as the chaotic behavior of the null geodesics. We can observe qualitatively different chaotic features in the dihole shadow [24].

Our findings indicate that the existence of the second compact object can affect gravitational wave emission from a test particle orbiting a main supermassive black hole because the inspiral phase tends to be longer than the case of a single black hole because of the shift of the ISCO radius. In the context of the quasinormal mode, the frequency 


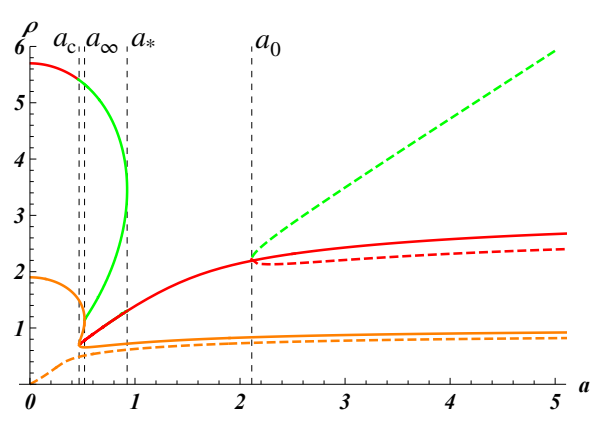

(a) $\nu=0.9$

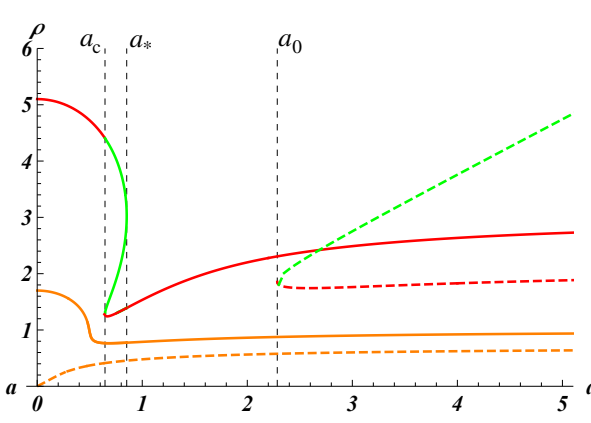

(b) $\nu=0.7$

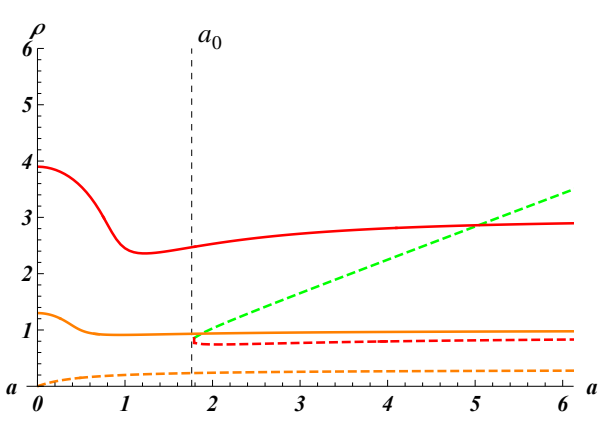

(c) $\nu=0.3$
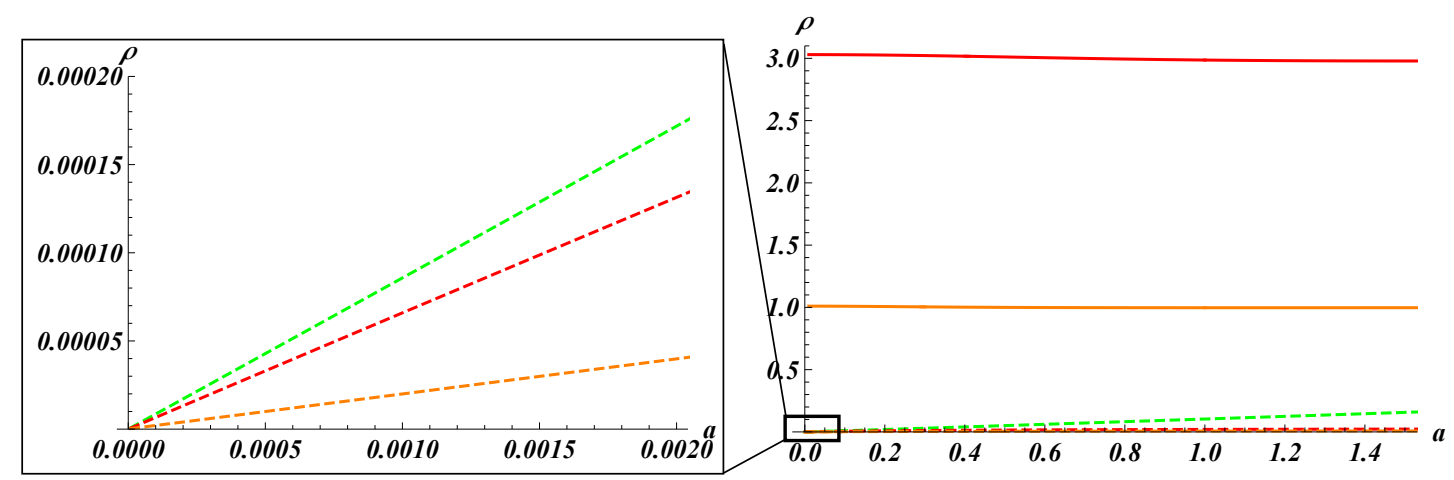

(d) $\nu=0.01$

FIG. 6: Dependence of the radii of MSCOs and circular photon orbits on the separation parameter a in the MP dihole spacetime with mass ratio: (a) $\nu=0.9$, (b) $\nu=0.7$, (c) $\nu=0.3$, and (d) $\nu=0.01$. The green and red solid lines show the radii of MSCOs on the large black hole side, and the green and red dashed lines show those on the small black hole side. In particular, the red lines indicate each ISCO. The orange solid lines show the radii of circular photon orbits on the large black hole side, and the orange dashed lines show those on the small black hole side. The dashed green and red lines merge at $a=a_{0}$ and then the sequence of the stable circular orbits on the small black hole side disappears. In cases (a) and (b), the green solid lines emerge at $a=a_{*}$, and the outer exist in the range $a_{\mathrm{c}}<a<a_{*}$. The inner in case (a) exists in the range $a_{\infty}<a<a_{*}$ while the one in case (b) exists in the range $a_{\mathrm{c}}<a<a_{*}$. In case (a), the stable circular photon orbits appear on the large black hole side in $a_{\mathrm{c}}<a<a_{\infty}$ whereas they do not in the other cases. In case (d), the sequence on the small black hole side always exists because there is no critical value of $a$.

is known to correspond to the orbital frequency of the unstable circular photon orbit. Since our results show that the orbital frequency can be comparable to that of a circular massive particle orbit, we can expect that the resonant excitation of the quasinormal mode occurs [31].

The MP dihole spacetime we have used in the background is static, but a realistic binary system is a dynamical system. Therefore, we should take into account dynamical features in future work.

\section{Acknowledgments}

The authors thank T. Harada, M. Kimura, T. Kobayashi, Y. Koga, and Y. Mizuno for their fruitful discussions and useful comments. This work was supported by a Grant-in-Aid for Early-Career Scientists from the Japan Society for the Promotion of Science (JSPS KAKENHI Grant No. JP19K14715) (T.I.) and the Rikkyo University Special Fund for Research (K.N.). K.N. also thanks the Yukawa Institute for Theoretical Physics at Kyoto University, where this 


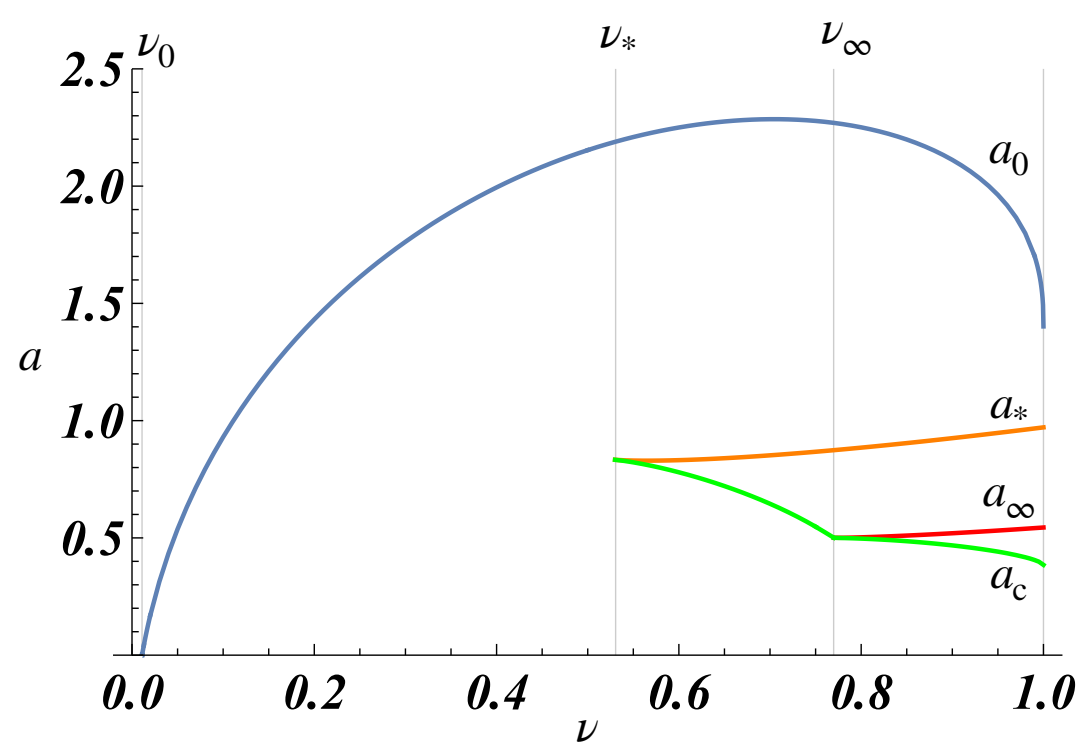

FIG. 7: Dependence of critical values of the separation $a$ on the mass ratio $\nu$. The blue, orange, red, and green lines show $a=a_{0}, a_{*}, a_{\infty}$, and $a_{\mathrm{c}}$, respectively. The parameter range of $\nu$ is divided into four parts, and the appearance of the critical values changes drastically at the boundaries, $\nu=\nu_{\infty}, \nu_{*}$, and $\nu_{0}$. At $\nu=\nu_{\infty}$, the critical values $a_{\infty}$ and $a_{\mathrm{c}}$ degenerate each other. At $\nu=\nu_{*}$, the critical values $a_{*}$ and $a_{\mathrm{c}}$ coincide with each other. At $\nu=\nu_{0}$, the critical value $a_{0}$ disappears.

work was developed during the "3rd Workshop on Gravity and Cosmology by Young Researchers" (YITP-W-18-15).

[1] B. P. Abbott et al. (LIGO Scientific and Virgo Collaborations), Observation of Gravitational Waves from a Binary Black Hole Merger, Phys. Rev. Lett. 116, 061102 (2016) [arXiv:1602.03837 [gr-qc]] [Search INSPIRE].

[2] B. P. Abbott et al. (LIGO Scientific and Virgo Collaborations), Properties of the Binary Black Hole Merger GW150914, Phys. Rev. Lett. 116, 241102 (2016) [arXiv:1602.03840 [gr-qc]] [Search INSPIRE].

[3] B. P. Abbott et al., Multi-messenger observations of a binary neutron star merger, Astrophys. J. 848, L12 (2017) [arXiv:1710.05833 [astro-ph.HE]] [Search INSPIRE].

[4] B. P. Abbott et al. [LIGO Scientific and Virgo Collaborations], GWTC-1: A Gravitational-Wave Transient Catalog of Compact Binary Mergers Observed by LIGO and Virgo During the First and Second Observing Runs, Phys. Rev. X 9, 031040 (2019) [arXiv:1811.12907 [astro-ph.HE]] [Search INSPIRE].

[5] K. Yamada and H. Asada, Collinear solution to the general relativistic three-body problem, Phys. Rev. D 82, 104019 (2010) [arXiv:1010.2284 [gr-qc]] [Search INSPIRE].

[6] K. Yamada and T. Tsuchiya, The linear stability of the post-Newtonian triangular equilibrium in the three-body problem, Celest. Mech. Dyn. Astr. 129, 487 (2017). [arXiv:1612.08361 [gr-qc]] [Search INSPIRE].

[7] N. Seto, Relativistic resonant relations between massive black hole binary and extreme mass ratio inspiral, Phys. Rev. D 85, 064037 (2012) [arXiv:1202.4761 [astro-ph.CO]][Search INSPIRE].

[8] H. Suzuki, P. Gupta, H. Okawa, and K. i. Maeda, Cumulative shift of periastron time of binary pulsar with Kozai-Lidov oscillation, Mon. Not. R. Astron. Soc. 486, L52 (2019) [arXiv:1903.00287 [gr-qc]][Search INSPIRE].

[9] L. Wen, On the eccentricity distribution of coalescing black hole binaries driven by the Kozai mechanism in globular clusters, Astrophys. J. 598, 419 (2003) [arXiv:astro-ph/0211492] [Search INSPIRE].

[10] N. Seto, Highly Eccentric Kozai Mechanism and Gravitational-Wave Observation for Neutron Star Binaries, Phys. Rev. Lett. 111,061106 (2013) [arXiv:1304.5151 [astro-ph.CO]] [Search INSPIRE].

[11] Y. Meiron, B. Kocsis, and A. Loeb, Detecting triple systems with gravitational wave observations, Astrophys. J. 834, 200 (2017) [arXiv:1604.02148 [astro-ph.HE]] [Search INSPIRE].

[12] J. P. A. Clark and D. M. Eardley, Evolution of close neutron star binaries, Astrophys. J. 215, 311 (1977).

[13] L. E. Kidder, C. M. Will, and A. G. Wiseman, Coalescing binary systems of compact objects to (post) ${ }^{5 / 2}$-Newtonian order. III. Transition from inspiral to plunge, Phys. Rev. D 47, 3281 (1993) [Search INSPIRE].

[14] A. Bohn, W. Throwe, F. Hébert, K. Henriksson, D. Bunandar, M. A. Scheel, and N. W. Taylor, What does a binary black hole merger look like?, Classical Quamtum Gravity 32, 065002 (2015) [arXiv:1410.7775 [gr-qc]] [Search INSPIRE].

[15] H. Weyl, The theory of gravitation, Ann. Phys. (N.Y.) 359, 117 (1917) [Search INSPIRE].

[16] S. D. Majumdar, A class of exact solutions of Einstein's field equations, Phys. Rev. 72, 390 (1947) [Search INSPIRE]. 
[17] A. Papaetrou, A static solution of the equations of the gravitational field for an arbitrary charge distribution, Proc. R. Ir. Acad., Sect. A 51, 191 (1947) [Search INSPIRE].

[18] J. B. Hartle and S. W. Hawking, Solutions of the Einstein-Maxwell equations with many black holes, Commun. Math. Phys. 26, 87 (1972) [Search INSPIRE].

[19] D. Kramer and G. Neugebauer, The superposition of two Kerr solutions, Phys. Lett. A 75, 259 (1980).

[20] D. Nitta, T. Chiba, and N. Sugiyama, Shadows of colliding black holes, Phys. Rev. D 84, 063008 (2011) [arXiv:1106.2425 [gr-qc]] [Search INSPIRE].

[21] M. Patil, P. Mishra, and D. Narasimha, Curious case of gravitational lensing by binary black holes: A tale of two photon spheres, new relativistic images and caustics, Phys. Rev. D 95, 024026 (2017) [arXiv:1610.04863 [gr-qc]] [Search INSPIRE].

[22] T. Assumpcao, V. Cardoso, A. Ishibashi, M. Richartz, and M. Zilhao, Black hole binaries: Ergoregions, photon surfaces, wave scattering, and quasinormal modes, Phys. Rev. D 98, 064036 (2018) [arXiv:1806.07909 [gr-qc]] [Search INSPIRE].

[23] P. V. P. Cunha, C. A. R. Herdeiro, and M. J. Rodriguez, Shadows of exact binary black holes, Phys. Rev. D 98, 044053 (2018) [arXiv:1805.03798 [gr-qc]] [Search INSPIRE].

[24] J. Shipley and S. R. Dolan, Binary black hole shadows, chaotic scattering and the Cantor set, Classical Quantum Gravity 33, 175001 (2016) [arXiv:1603.04469 [gr-qc]] [Search INSPIRE].

[25] K. Nakashi and T. Igata, Innermost stable circular orbits in the Majumdar-Papapetrou dihole spacetime, Phys. Rev. D 99, 124033 (2019) [arXiv:1903.10121 [gr-qc]] [Search INSPIRE].

[26] T. Ono, T. Suzuki, and H. Asada, Nonradial stability of marginal stable circular orbits in stationary axisymmetric spacetimes, Phys. Rev. D 94, 064042 (2016) [arXiv:1605.05816 [gr-qc]] [Search INSPIRE].

[27] A. Wunsch, T. Muller, D. Weiskopf, and G. Wunner, Circular orbits in the extreme Reissner-Nordstrøm dihole metric, Phys. Rev. D 87, 024007 (2013) [arXiv:1301.7560 [gr-qc]] [Search INSPIRE].

[28] I. D. Novikov and K. S. Thorne, Astrophysics of black holes, in Black Holes (Les Astres Occus), edited by C. DeWitte and B. DeWitt (Gordon and Breach, New York, 1973), p. 343.

[29] D. N. Page and K. S. Thorne, Disk-accretion onto a black hole. Time-averaged structure of accretion disk, Astrophys. J. 191, 499 (1974) [Search INSPIRE].

[30] M. J. Valtonen et al., A massive binary black-hole system in OJ 287 and a test of general relativity, Nature (London) 452, 851 (2008) [arXiv:0809.1280 [astro-ph]] [Search INSPIRE].

[31] L. Bernard, V. Cardoso, T. Ikeda, and M. Zilhão, Physics of black hole binaries: Geodesics, relaxation modes, and energy extraction, Phys. Rev. D 100, 044002 (2019) [arXiv:1905.05204 [gr-qc]] [Search INSPIRE]. 